\title{
Phenomenology of ripples in graphene
}

\author{
S. A. Ktitorov ${ }^{1,2}$, R. I. Mukhamadiarov ${ }^{1}$ \\ ${ }^{1}$ Ioffe Institute, St. Petersburg, Russia, \\ ${ }^{2}$ St. Petersburg Electrotechnical University LETI, St. Petersburg, Russia \\ ktitorov@mail.ioffe.ru
}

\section{PACS 68.65.Pq}

DOI 10.17586/2220-8054-2016-7-1-58-59

\begin{abstract}
The ripples are considered an incommensurate superstructure in a two-dimensional crystal, appearing as a result of the formation of periodic solutions in an in-plane optical phonon subsystem. The possible instability of the flexural subsystem is also discussed.
\end{abstract}

Keywords: Landau functional, graphene, ripples, incommensurate, curvature.

Received: 20 November 2015

\section{Introduction}

A recent experimental study has shown that the flat geometry of graphene is unstable, leading to the formation of a corrugated structure: topological defects and ripples [1]. Graphene can be viewed as a crystalline membrane. An ideal flat 2D-crystal could not exist at a finite temperature [1], and the long range order in graphene's structure occurs because of ripples and topological defects. Both of these factors facilitate the achievement of thermodynamic stability in grapheme [2] and they could be induced to relieve the membrane's strain. As far as we know, no widely accepted model for ripples in monolayer graphene exists. The crumpled membrane theory does not work for graphene at relevant temperatures. Some authors try to obtain a ripplelike solution from the renormalization group approach, but the only result is the appearance of some characteristic length of a correct magnitude. The phenomenon under consideration looks too simple to be described with a use of the sophisticated fluctuation theory: we assume that the ripple theory has to be the mean field one. The somewhat similar problem of undulations in biological membranes was solved by taking into account the internal degrees of freedom [3]. One such fluctuating degree of freedom was the membrane thickness. In the case of graphene, a natural candidate on this position is the in-plane transverse optical phonon. The spatial period of the ripple is determined by the ratio of the coefficients in the thermodynamic potential derivative expansion. The out-of-plane subsystem acquires periodicity due to the interaction between the subsystems. Two interaction mechanisms: bilinear and biquadratic were considered. Only solutions with vanishing Gaussian curvature are admissible. The suggested phenomenological description needs to be justified by future microscopic theory.

\section{In-plane phonon instability}

The equilibrium state of the membrane is determined by the effective potential in the Monge representation [3]:

$$
F[h]=\int d^{2} x\left[\frac{\kappa}{2}\left(\nabla^{2} h\right)^{2}+\frac{K_{0}}{8}\left[P_{\alpha \beta}\left(\partial_{\alpha} h \cdot \partial_{\beta} h\right)\right]^{2}\right],
$$

where $\kappa$ is the bending rigidity, $h$ is the membrane height relative to the base plane (Monge variable), and the second term is Gaussian curvature. 


$$
P_{\alpha \beta}^{T}=\delta_{\alpha \beta}-\frac{q_{\alpha} q_{\beta}}{q^{2}}, \quad K=\frac{2 \mu(2 \mu+D \lambda)}{2 \mu+\lambda},
$$

$P_{\alpha \beta}$ is normal projector, $\mu$ and $\lambda$ are the Lame elastic moduli. Here, we assume this subsystem to be stable in isolation. In our case, the natural candidate on the symmetry violating system position is the in-plane transverse optical phonon. The simplest Landau functional containing a certain spatial scale (apart from the lattice spacing) in a crystal without the Lifshitz invariant is the one suggested for incommensurate ferroelectrics [4]:

$$
F_{\text {incomm }}=\int d x d y\left[\frac{a_{2}}{2} \varphi^{2}+\frac{a_{4}}{4} \varphi^{4}+\frac{c_{2}}{2}(\nabla \varphi)^{2}+\frac{d_{2}}{2}\left(\nabla^{2} \varphi\right)^{2}\right] .
$$

Here, $a_{2}<0, a_{4}>0, c>0, d>0$. Varying this functional, we obtain the equilibrium equation:

$$
d_{2} \frac{d^{4} \varphi}{d x^{4}}+c_{2} \frac{d^{2} \varphi}{d x^{2}}+\left|a_{2}\right| \varphi-a_{4} \varphi^{3}=0 .
$$

We consider here unidimensional solutions. For the chosen signature of the coefficients, there exists a periodic solution with the characteristic period value of order $\sqrt{d_{2} / c_{2}}$. This violation of the translation symmetry can be transferred to the out-of-plane subsystem by means of an interaction. The simplest interactions that do not break the spatial inversion symmetry read:

$$
g \int d x d y \varphi \Delta_{2} h
$$

and

$$
G \int d x d y \varphi^{2}\left(\Delta_{2}^{2} h\right)^{2}
$$

Interaction (5) is more effective, but is not easy to derive. Now, we consider another scenario.

\section{Out-plane phonon instability}

Corresponding with the Landau-Peierls-Mermin-Wagner theorem, the logarithmic divergencies indicating the instability of the flat state are characteristic for two-dimensional systems. In particular, the bending rigidity constant will be renormalized [3]:

$$
\kappa_{r}=\kappa_{0}-\frac{3 k_{B} T}{4 \pi} \ln \frac{L}{a} .
$$

Here, $a$ is the lattice spacing and $L$ is the sample size. The possible change of sign in (7) induces an instability similar to the case considered above. We believe that fluctuations can make absolute value negative. This may induce instability, which stabilizes by non-linear terms as in phase transitions theory.

\section{References}

[1] Vozmediano M.A.H., Katsnelson M.I., Guinea F. Gauge fields in graphene. Physics Reports, 2010, 496, P. 109-148.

[2] Mermin N.D., Wagner H. Absence of Ferromagnetism or Antiferromagnetism in One- or Two-Dimensional Isotropic Heisenberg Models. Phys. Rev. Lett., 1966, 17, P. 1133-1136.

[3] Nelson D., Piran T., Weinberg S. Statistical Mechanics of Membranes Surfaces, World Scientific, New Jersey, 2004.

[4] Izyumov Y.A., Syromyatnikov V.N. Phase transitions and symmetry of crystals. Science, Moscow, 1984, $182 \mathrm{p}$. 